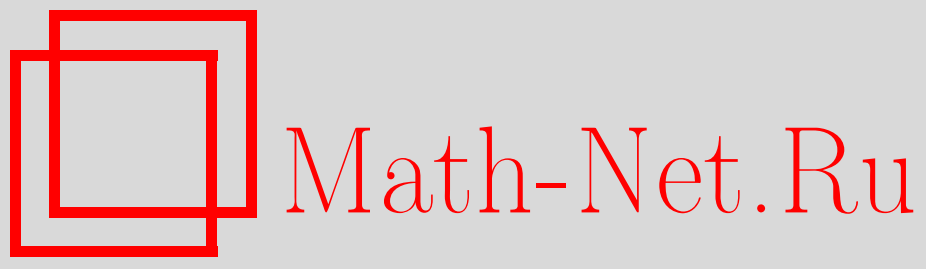

С. А. Степин, О модели Фридрихса в односкоростной теории переноса, Функи. анализ и его прил., 2001, том 35, выпуск 2, 87-92

DOI: https://doi.org/10.4213/faa252

Использование Общероссийского математического портала MathNet.Ru подразумевает, что вы прочитали и согласны с пользовательским соглашением

http://www . mathnet.ru/rus/agreement

Параметры загрузки:

IP: 54.237 .206 .68

26 апреля 2023 г., 15:16:06

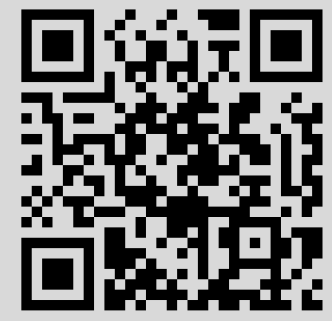


$B_{i} \cap C-$ непустые множества, то $A_{i} \cap C=C=B_{i} \cap C$, так как $C$ является атомом. Но это противоречит условию $A_{i} \cap B_{i}=\varnothing$. Таким образом, $C \times C$ не пересекается с объединением множеств $A_{i} \times B_{i}$. Всегда найдется такой атом $C$, что $\mu \otimes \mu(C \times C) \geqslant 2^{-4 r}$.

\title{
ЛИТЕРАТУРА
}

1. Вершик A. М. Записки научных семинаров ЛОМИ, 72, 26-62 (1977). 2. Ferenczi S. Colloquium Mathematicum, 73, No. 1, 35-65 (1997). 3. Goodson G. R., Ryzhikov V. $V$. J. Dynamical and Control Systems, 3, 321-341 (1997). 4. Katok A. Constructions in ergodic theory. Preprint. 5. Оселедец В. И. Матем. заметки, 5, №3, 323-326 (1969). 6. Рыжиков В. В. Матем. сб., 183, №3, 133-160 (1992). 7. Степин А. М. Изв. АН СССР, сер. матем., 50, 801-834 (1986).

Московский государственный университет, механико-математический факультет vryz@mech.math.msu.su

Поступило в редакцию 31 января 2000 г.

УДК 517.9

\section{О модели Фридрихса в односкоростной теории переноса*}

\author{
(c) 2001. С. А. Степин
}

В настоящей работе изучаются спектральные свойства несамосопряженного интегро-дифференциального оператора, возникающего при линеаризации уравнения Больцмана в рамках односкоростной модели переноса частиц в веществе (см. [1]). Рассматривается случай, когда количественные характеристики рассеяния частиц зависят лишь от одной пространственной координаты, скажем $x$. Процесс рассеяния описывается функцией распределения $\Psi(x, \mu, t)$, имеющей смысл плотности числа частиц, находящихся в момент времени $t$ в точке с координатой $x$ и таких, что косинус угла между скоростью частицы и осью $x$ равен $\mu$. Линеаризованное уравнение эволюции функции распределения имеет вид

$$
\left(\frac{1}{v} \frac{\partial}{\partial t}+\mu \frac{\partial}{\partial x}+\sigma\right) \Psi(x, \mu, t)=\sigma \frac{c(x)}{2} \int_{-1}^{1} \Psi(x, \mu, t) d \mu .
$$

Здесь $v$ - скорость частиц, $\sigma$ - макроскопическое сечение (величина, обратно пропорциональная средней длине свободного пробега частиц), а $c(x)$ - коэффициент размножения, характеризующий взаимодействие частиц с веществом. Предполагается, что $c(x)$ - неотрицательная (существенно) ограниченная функция.

В этом контексте К. О. Фридрихсом была поставлена (см. $[2,4])$ задача изучения спектральных свойств действующего в пространстве $\mathscr{H}=\mathrm{L}^{2}(\mathbb{R} \times[-1,1])$ оператора

$$
L=L_{0}+i V=i \mu \frac{\partial}{\partial x}+i \frac{c(x)}{2} \int_{-1}^{1} \cdot d \mu
$$

* Работа выполнена при поддержке Российского фонда фундаментальных исследований. 
с областью определения $D(L)=\{\psi \in \mathscr{H}: \psi(\cdot, \mu)$ абсолютно непрерывна для п. в. $\mu \in[-1,1], L \psi \in \mathscr{H}\}$. В работах $[3,4]$ был исследован спектр оператора $L$ в случае, когда функция $c(x)$ пропорциональна индикатору интервала. Случай произвольной финитной неотрицательной функции $c(x) \in \mathrm{L}^{\infty}(\mathbb{R})$ изучен в [5]. Для таких $c(x)$ непрерывный спектр оператора $L$ заполняет вещественную ось, а точечный спектр состоит из нормальных полупростых собственных значений, принадлежащих $i \mathbb{R}_{+}$. В работе [5] для суммарной кратности $N(c)$ собственных значений оператора $L$ в случае финитной функции $c(x)$ получена оценка

$$
N(c) \leqslant 1+\frac{1}{4} \iint_{\mathbb{R} \times \mathbb{R}} c(x)(\ln |x-y|)^{2} c(y) d x d y .
$$

В этой связи возникает вопрос, будет ли оценка (1) верна при одном лишь условии конечности ее правой части. В настоящей работе получен положительный ответ на этот вопрос. Кроме того, показано, что в случае, когда $c(x) \in$ $\mathrm{L}^{\infty}(\mathbb{R}) \cap \mathrm{L}^{2}(\mathbb{R})$, у оператора $L$ сохраняется указанная выше структура спектра, а также изучена величина $N(\kappa c)$ как функция параметра $\kappa>0$.

В дальнейшем используются следующие обозначения: $\sigma(A)$ - спектр оператора $A, \sigma_{c}(A)$ и $\sigma_{p}(A)$ - непрерывная и точечная компоненты спектра $\sigma(A)$, $R(\lambda):=(L-\lambda I)^{-1}$ и $R_{0}(\lambda):=\left(L_{0}-\lambda I\right)^{-1}$.

1. Структура спектра оператора $\boldsymbol{L}$. Применительно к рассматриваемой здесь ситуации включения $\mathbb{R} \subset \sigma_{c}(L)$ и $\sigma_{p}(L) \subset i \mathbb{R}_{+}$устанавливаются так же, как в работе [4]. Поскольку оператор $V \geqslant 0$ ограничен, то $L=L_{0}+i V-$ максимальный диссипативный оператор и, следовательно, $\sigma(L) \cap \mathbb{C}_{-}=\varnothing$.

Для $\lambda \in \mathbb{C}_{+} \backslash \sigma(L)$ справедливо следующее тождество (см. $\left.[4,5]\right)$ :

$$
R(\lambda)=R_{0}(\lambda)-i R_{0}(\lambda) \sqrt{V}(I+Q(\lambda))^{-1} \sqrt{V} R_{0}(\lambda),
$$

где $Q(\lambda)-$ интегральный оператор с ядром

$$
q(x, y ; \lambda):=\frac{1}{2} \sqrt{c(x)} \operatorname{Ei}(i \lambda|x-y|) \sqrt{c(y)}
$$

здесь $\operatorname{Ei}(z)$ - интегральная показательная функция,

$$
\operatorname{Ei}(z)=-\int_{1}^{\infty} \frac{e^{z t}}{t} d t, \quad \operatorname{Re} z<0 .
$$

Допустим, что $[Q(\lambda)]^{l}$ при некотором $l \in \mathbb{N}$ и всех $\lambda \in \mathbb{C}_{+}$принимает значения в классе $\mathfrak{S}_{\infty}$ компактных операторов. Тогда, согласно аналитической альтернативе Фредгольма, оператор-функция $(I+Q(\lambda))^{-1}$ мероморфна в $\mathbb{C}_{+}$, причем ее вычеты в полюсах суть операторы конечного ранга. Ввиду соотношения (2) то же самое верно для резольвенты $R(\lambda)$, и потому часть спектра оператора $L$, содержащаяся в открытой верхней полуплоскости, представляет собой дискретное множество конечнократных собственных значений.

Следующее утверждение дает достаточное условие компактности оператора $Q(\lambda), \lambda \in \mathbb{C}_{+}$, и, сверх того, принадлежности $Q(\lambda)$ классу $\mathfrak{S}_{2}$ операторов Гильберта-Шмидта.

ПрЕДЛОЖЕНИЕ 1. Если $c(x) \in \mathrm{L}^{\infty}(\mathbb{R}) \cap \mathrm{L}^{1}(\mathbb{R})$ u $c(x) \geqslant 0$ при п. в. $x \in \mathbb{R}$, mо $Q(\lambda) \in \mathfrak{S}_{2}$ для каждого $\lambda=\alpha+i \beta \in \mathbb{C}_{+}$. 
ДоКАЗАТЕЛЬСтво. Из формулы (3) при $\beta|x-y| \geqslant 1$ следует неравенство $|q(x, y ; \lambda)| \leqslant \sqrt{c(x) c(y)}$. В случае если $T:=\alpha|x-y| \geqslant 1$, имеем

$\operatorname{Ei}(i \lambda|x-y|)=-\int_{T}^{\infty} \frac{e^{-\varepsilon t}}{t} e^{i t} d t=-e^{-\varepsilon T} \int_{T}^{\infty} \frac{e^{i t}}{t} d t+\varepsilon \int_{T}^{\infty} e^{-\varepsilon t}\left(\int_{t}^{\infty} \frac{e^{i \tau}}{\tau} d \tau\right) d t$, где $\varepsilon=\beta / \alpha$. Отсюда видно, что

$$
|\operatorname{Ei}(i \lambda|x-y|)| \leqslant 2 \max _{t \geqslant 1}\left|\int_{t}^{\infty} \frac{e^{i \tau}}{\tau} d \tau\right|,
$$

когда $|\alpha||x-y| \geqslant 1$. Таким образом,

$$
|q(x, y ; \lambda)| \leqslant M \sqrt{c(x) c(y)}
$$

если $|x-y| \geqslant \delta=\min \left\{|\alpha|^{-1}, \beta^{-1}\right\}$, где постоянная $M$ больше или равна 1 .

Рассмотрим теперь случай, когда $|x-y| \leqslant \delta$. Используя известное представление интегральной экспоненты

$$
\operatorname{Ei}(z)=\ln (-z)+\gamma+\sum_{n=1}^{\infty} \frac{z^{n}}{n \cdot n !}
$$

и учитывая, что $|\lambda||x-y| \leqslant 2$ и $|\arg (-i \lambda)| \leqslant \pi / 2$, получаем неравенство

$$
|q(x, y ; \lambda)|=\frac{1}{2} \sqrt{c(x)}|\operatorname{Ei}(i \lambda|x-y|)| \sqrt{c(y)} \leqslant \frac{1}{2} \sqrt{c(x)}(|\ln | x-y||+K(\lambda)) \sqrt{c(y)},
$$

где $K(\lambda)=|\ln | \lambda||+$ const. Сказанное позволяет утверждать, что при сделанных относительно коэффициента $c(x)$ предположениях для $\lambda \in \mathbb{C}_{+}$ядро $q(x, y ; \lambda)$ квадратично интегрируемо и, стало быть, $Q(\lambda)$ принадлежит классу $\mathfrak{S}_{2}$.

TEOPEMA 1. Ecлu $c(x) \in \mathrm{L}^{\infty}(\mathbb{R}) \cap \mathrm{L}^{2}(\mathbb{R})$ u $c(x) \geqslant 0$ nрu n. в. $x \in \mathbb{R}$, mо иасть спектра $\sigma(L) \cap \mathbb{C}_{+} \subset i \mathbb{R}_{+}$состоит из изолированных собственных значений конечной кратности.

Действительно, если $0 \leqslant c(x) \in \mathrm{L}^{\infty}(\mathbb{R}) \cap \mathrm{L}^{2}(\mathbb{R})$, то в силу предложения 1 оператор $V R_{0}(\lambda) V$ принадлежит $\mathfrak{S}_{\infty}$ для всех $\lambda \in \mathbb{C}_{+}$. Отсюда с учетом тождества

$$
\operatorname{Im}\left(V R_{0}(\lambda) V\right)=\operatorname{Im} \lambda V R_{0}(\lambda)\left(V R_{0}(\lambda)\right)^{*}
$$

заключаем, что $V R_{0}(\lambda) \in \mathfrak{S}_{\infty}$ и, следовательно,

$$
Q^{2}(\lambda)=-\sqrt{V} R_{0}(\lambda) V R_{0}(\lambda) \sqrt{V} \in \mathfrak{S}_{\infty}, \quad \lambda \in \mathbb{C}_{+} .
$$

Отметим, что все собственные значения оператора $L$ полупростые, т.е. соответствующие корневые подпространства не содержат присоединенных векторов.

2. Условие конечности дискретного спектра оператора $\boldsymbol{L}$. Обозначим через $N_{\varepsilon}(c), \varepsilon>0$, ранг спектрального проектора оператора $L$, отвечающего части спектра, принадлежащей промежутку $[i \varepsilon, i \infty)$. Так как $\operatorname{dim} \operatorname{ker}(L-\lambda I)=$ $\operatorname{dim} \operatorname{ker}(I+Q(\lambda))$, то

$$
N_{\varepsilon}(c)=\sum \operatorname{dim} \operatorname{ker}(I+Q(i s)),
$$

где суммирование ведется по тем $s \geqslant \varepsilon$, для которых $-1 \in \sigma_{p}(Q(i s))$. Отметим, что собственные значения компактного самосопряженного оператора $Q(i s) \leqslant 0$ непрерывно зависят от $s$ и, поскольку $d Q(i s) / d s \geqslant 0$, они движутся вправо с ростом параметра $s$. При этом $\|Q(i s)\| \rightarrow 0$, если $s \rightarrow \infty$, и, следовательно, 
$(-\infty,-1] \cap \sigma(Q(i s))=\varnothing$ для достаточно больших значений $s$. Таким образом, устанавливается

ПреДЛОЖенИЕ 2. Пусть $c(x) \in \mathrm{L}^{\infty}(\mathbb{R})$ u $c(x) \geqslant 0$ при п. в. $x \in \mathbb{R}$. Eсли $Q(\lambda) \in$ $\mathfrak{S}_{\infty}, \lambda \in \mathbb{C}_{+}$, то $N_{\varepsilon}(c)$ совпадает с суммарной кратностью собственных значений оператора $Q(i \varepsilon)$, принадлежащих $(-\infty,-1]$.

Способ подсчета количества собственных значений оператора Шрёдингера, основанный на тех же соображениях, что и выше, известен под названием принципа Бирмана-Швингера (см. [6]). В работе [5] подобные соображения использовались для получения оценки суммарной кратности собственных значений оператора $L$ в случае финитной функции $c(x) \in \mathrm{L}^{\infty}(\mathbb{R})$.

TEOPEMA 2. Пусть $c(x) \in \mathrm{L}^{\infty}(\mathbb{R}), c(x)(\ln |x-y|)^{2} c(y) \in \mathrm{L}^{1}(\mathbb{R} \times \mathbb{R}) u c(x) \geqslant 0$ при п.в. $x \in \mathbb{R}$. Тогда точечный спектр $\sigma_{p}(L)$ конечен и суммарная кратность $N(c)$ собственных значений оператора L допускает оценку

$$
N(c) \leqslant 1+\frac{1}{4} \iint_{\mathbb{R} \times \mathbb{R}} c(x)(\ln |x-y|)^{2} c(y) d x d y .
$$

ДокАЗАТЕЛЬСтво. В предположениях теоремы имеем $c(x) \in \mathrm{L}^{1}(\mathbb{R})$ и, стало быть, $N_{\varepsilon}(c)<\infty$. Обозначим через $P_{\varepsilon}$ оператор с вырожденным ядром $\sqrt{c(x) c(y)}(\gamma+\ln \varepsilon) / 2$ и отметим, что ядро $q(x, y ; i \varepsilon)$ интегрального оператора $Q(i \varepsilon), \varepsilon>0$, представимо в виде

$$
\begin{aligned}
q(x, y ; i \varepsilon)=\frac{1}{2} \sqrt{c(x)} & \ln |x-y| \sqrt{c(y)} \\
& +\frac{1}{2}(\gamma+\ln \varepsilon) \sqrt{c(x) c(y)}-\frac{1}{2} \sqrt{c(x)} \operatorname{Ein}(\varepsilon|x-y|) \sqrt{c(y)},
\end{aligned}
$$

где $\operatorname{Ein}(z)-$ дополнительная интегральная показательная функция:

$$
\operatorname{Ein}(z)=\int_{0}^{z} \frac{1-e^{-t}}{t} d t .
$$

Используя предложение 2 и учитывая, что $\operatorname{rank} P_{\varepsilon}=1$, согласно теореме Вейля и Куранта о сравнении собственных значений, получаем

$$
N_{\varepsilon}(c)-1 \leqslant \sum_{s \leqslant-1} \operatorname{dim} \operatorname{ker}\left[Q(i \varepsilon)-P_{\varepsilon}-s I\right] \leqslant\left\|Q(i \varepsilon)-P_{\varepsilon}\right\|_{\mathfrak{S}_{2}}^{2} .
$$

Вследствие этой оценки и представления (4)

$$
\begin{aligned}
& N_{\varepsilon}(c) \leqslant 1+\frac{1}{4}\left\{\left(\iint_{\mathbb{R} \times \mathbb{R}} c(x)(\ln |x-y|)^{2} c(y) d x d y\right)^{1 / 2}\right. \\
&\left.+\left(\iint_{\mathbb{R} \times \mathbb{R}} c(x)(\operatorname{Ein}(\varepsilon|x-y|))^{2} c(y) d x d y\right)^{1 / 2}\right\}^{2} .
\end{aligned}
$$

Поскольку $\operatorname{Ein}(s) \leqslant s$ при $s>0$, справедливы неравенства

$$
\begin{gathered}
\iint_{\varepsilon|x-y|<\delta} c(x)(\operatorname{Ein}(\varepsilon|x-y|))^{2} c(y) d x d y \leqslant \delta^{2}\left(\int_{\mathbb{R}} c(x) d x\right)^{2}, \\
\iint_{\delta \leqslant \varepsilon|x-y| \leqslant 1} c(x)(\operatorname{Ein}(\varepsilon|x-y|))^{2} c(y) d x d y \leqslant \iint_{\varepsilon|x-y| \geqslant \delta} c(x) c(y) d x d y
\end{gathered}
$$


с произвольным $\delta>0$, откуда видно, что

$$
\iint_{\varepsilon|x-y| \leqslant 1} c(x)(\operatorname{Ein}(\varepsilon|x-y|))^{2} c(y) d x d y \rightarrow 0, \quad \varepsilon \downarrow 0 .
$$

Далее, при $s \geqslant 1$ имеем $\operatorname{Ein}(s) \leqslant 1+\ln s$ и, следовательно,

$\iint_{\varepsilon|x-y| \geqslant 1} c(x)(\operatorname{Ein}(\varepsilon|x-y|))^{2} c(y) d x d y \leqslant \iint_{\varepsilon|x-y| \geqslant 1} c(x)(1+\ln (\varepsilon|x-y|))^{2} c(y) d x d y$.

Так как $c(x)(\ln |x-y|)^{2} c(y) \in \mathrm{L}^{1}(\mathbb{R} \times \mathbb{R})$, то

$$
\iint_{\varepsilon|x-y| \geqslant 1} c(x)(\operatorname{Ein}(\varepsilon|x-y|))^{2} c(y) d x d y \rightarrow 0, \quad \varepsilon \downarrow 0 .
$$

Переходя к пределу в (5) при $\varepsilon \downarrow 0$, получаем искомую оценку для $N(c)$.

3. Рождение собственных значений. Рассмотрим однопараметрическое семейство операторов $L(\kappa):=L_{0}+i \kappa V, \kappa \in \mathbb{R}_{+}$, где функция $c(x)$ удовлетворяет требованиям теоремы 2 , и отметим, что величина $N(\kappa c)$ монотонно возрастает с ростом параметра $\kappa$. При достаточно малых значениях параметра $\kappa>0$ оператор $L(\kappa)$ имеет ровно одно собственное значение, если $c(x)>0$ на множестве положительной меры. При увеличении $\kappa$ появляются новые собственные значения, и механизм их возникновения может быть описан на основании предложения 2. Особую роль при этом играет подмножество $\mathscr{E} \subset \mathrm{L}^{\infty}(\mathbb{R}) \cap \mathrm{L}^{1}(\mathbb{R})$, выделенное следующим условием:

$$
0 \leqslant c(x) \in \mathscr{E} \Longleftrightarrow \liminf _{\varepsilon \downarrow 0} \operatorname{dist}\{-1, \sigma(Q(i \varepsilon))\}=0 .
$$

ПрЕДЛОЖЕНИЕ 3. Пусть $c(x)$ удовлетворяет условиям теоремь 2. Если $c(x) \notin \mathscr{E}$, то величина $N(\kappa c)$ постоянна в окрестности значения $\kappa=1$; если же $c(x) \in \mathscr{E}$, mo $N(\kappa c)>N(c)$ nрu $\kappa>1$.

ДоКАЗАТЕЛЬствО. Как уже отмечалось, собственные значения $\mu_{n}(s)$ оператора $Q(i s)$ представляют собой монотонные функции параметра $s$. Поэтому существуют конечные или бесконечные пределы $\mu_{n}(+0)$ и условие $(6)$ может быть записано в следующей форме: $-1 \in\left\{\mu_{n}(+0)\right\}_{n=1}^{\infty}$.

Если $c(x) \notin \mathscr{E}$, то $-1 \notin\left\{\mu_{n}(+0)\right\}_{n=1}^{\infty}$. Положим $N:=N(c)$; тогда $\mu_{N}(+0)<-1$ и $\mu_{N+1}(+0)>-1$. Согласно предложению 2 , для всех значений параметра $\kappa$, достаточно близких к 1 , имеем $N_{\varepsilon}(\kappa c)=N$, если $\varepsilon>0$ достаточно мало, и, следовательно,

$$
N(\kappa c)=\lim _{\varepsilon \downarrow 0} N_{\varepsilon}(\kappa c)=N,
$$

т. е. ранг спектрального проектора $N(\kappa c)$ постоянен в окрестности значения $\kappa=1$. Если же $c(x) \in \mathscr{E}$, то $\mu_{N+1}(+0)=-1$, причем $\mu_{N+1}(\varepsilon)>-1$ для $\varepsilon>0$, и $\mu_{N}(+0)<-1$. В силу предложения 2 для произвольного $\kappa>1$ справедливо неравенство $N_{\varepsilon}(\kappa c) \geqslant N+1$, если $\varepsilon>0$ достаточно мало, и, стало быть,

$$
N(\kappa c)=\lim _{\varepsilon \downarrow 0} N_{\varepsilon}(\kappa c)>N(c) .
$$

В случае $c(x) \in \mathscr{E}$ произвольному $\kappa>1$ отвечает число $\varepsilon_{0}(\kappa)>0$, такое, что $N_{\varepsilon}(\kappa c)>N(c)$, если $\varepsilon \in\left(0, \varepsilon_{0}\right)$, и $N_{\varepsilon}(\kappa c) \leqslant N(c)$ для $\varepsilon \geqslant \varepsilon_{0}$, причем $\varepsilon_{0}(\kappa) \rightarrow 0$, когда $\kappa \rightarrow 1$. Сказанное позволяет сделать следующий вывод: рождение новых 
собственных значений оператора $L(\kappa)$ с ростом $\kappa$ происходит из точки $\lambda=0$ при тех и только тех значениях параметра $\kappa$, для которых $\kappa c \in \mathscr{E}$.

\title{
ЛИТЕРАТУРА
}

1. Шихов С. Б. Вопросы математической теории реакторов. Линейный анализ. Атомиздат, M., 1973. 2. Pихтмайер P. Принципы современной математической физики. Мир, M., 1982. 3. Lehner J., Wing G. M. Comm. Pure Appl. Math., 8, 217-234 (1955). 4. Lehner J. J. Math. Mech., 11, No. 2, 173-181 (1962). 5. Куперин Ю. А., Набоко C. Н., Романов Р. В. Функц. анализ и его прил., 33, вып. 3, 47-58 (1999). 6. Simon B. Functional integration and quantum physics. Academic Press, New York, 1979.

Московский государственный университет, механико-математический факультет

Поступило в редакцию 6 января 2000 г.

УДК 517.919

\section{Некристаллографические группы Кокстера и краевые особенности}

\author{
(C) 2001. И. Г. ЩЕРБАК
}

о. Введение. В работе 1972 г. [1] В. И. Арнольд описал деформации голоморфных ростков функций, миниверсальные относительно стабильной эквивалентности, и показал, что для ростков типа $A_{\mu}, D_{\mu}, E_{6}, E_{7}, E_{8}$ соответствующие бифуркационные диаграммы диффеоморфны многообразиям нерегулярных орбит комплексифицированного действия соответствующих групп отражений.

Деформации тех же ростков, миниверсальные относительно других отношений эквивалентности, возникают естественным образом при изучении особенностей с дополнительными структурами, такими, как граница, препятствие, симметрии и т. д. Среди соответствующих бифуркационных диаграмм можно распознать многообразия нерегулярных орбит других групп Кокстера. Так, например, миниверсальные деформации простых особенностей функций на многообразии с краем отождествляются с деформациями ростков $A_{2 \mu-1}, D_{\mu+1}, E_{6}$, миниверсальными в классе $\mathbb{Z}_{2}$-симметричных функций, и соответствующие бифуркационные диаграммы диффеоморфны многообразиям нерегулярных орбит групп отражений $B_{\mu}, C_{\mu}, F_{4}$ [2]. Многообразия нерегулярных орбит групп Кокстера $I_{2}(5), H_{3}$, $H_{4}$ появляются в задаче об обходе препятствия как бифуркационные диаграммы деформаций особенностей $A_{4}, D_{6}, E_{8}$ соответственно, миниверсальных в классе четнократных особенностей [3].

В отличие от этих примеров, особенности, связанные с группами Кокстера $I_{2}(p)(p \geqslant 5)$ и $H_{3}$, появляются в теории Ляшко критических точек функций на многообразии с особым краем [4] в ином контексте. В настоящей заметке устанавливается связь особенностей Ляшко с краевыми особенностями. Многообразия нерегулярных орбит групп $I_{2}(p)$ и $H_{3}$ оказываются диффеоморфными бифуркационным диаграммам краевых деформаций простых особенностей $A_{3}$ и $A_{4}$, связанных с краевыми особенностями $B_{p-1}^{3}$ и $F_{4}^{4}$ соответственно. Таким образом, эти группы включаются в «стандартную» схему, описанную выше. Необходимые понятия и факты читатель может найти в работах $[2,4,5]$ (см. также книгу $[6]$ ). 